\title{
Linx
}

Revue des linguistes de l'université Paris X Nanterre

$7 \mid 1995$

Saussure aujourd'hui

\section{Réflexions saussuriennes sur le temps et le moi}

Les manuscrits de la Houghton Library à Harvard

\section{Herman Parret}

\section{CpenEdition}

\section{Journals}

Édition électronique

URL : http://journals.openedition.org/linx/1124

DOI : 10.4000/linx.1124

ISSN : 2118-9692

Éditeur

Presses universitaires de Paris Nanterre

Édition imprimée

Date de publication : 1 septembre 1995

Pagination : 39-74

ISSN : 0246-8743

\section{Référence électronique}

Herman Parret, « Réflexions saussuriennes sur le temps et le moi », Linx [En ligne], 7| 1995, mis en ligne le 12 juillet 2012, consulté le 10 décembre 2020. URL : http://journals.openedition.org/linx/1124 ; DOl : https://doi.org/10.4000/linx.1124

Ce document a été généré automatiquement le 10 décembre 2020.

Département de Sciences du langage, Université Paris Ouest 


\title{
Réflexions saussuriennes sur le temps et le moi
}

\author{
Les manuscrits de la Houghton Library à Harvard*
}

\author{
Herman Parret
}

1 La Houghton Library, section des manuscrits et des livres précieux de la Widener Library, bibliothèque de l'Université de Harvard (Cambridge, Massachusetts, Etats-Unis), a pu acheter en 1967 un ensemble de manuscrits de Ferdinand de Saussure au Docteur Raymond de Saussure. Cet achat a été rendu possible grâce à l'intervention de Roman Jakobson, professeur à Harvard et à M.I.T. à ce moment ${ }^{1}$. Jakobson mentionne ces manuscrits dans un article de 1969 où il formule ainsi leur importance :

" These newfound materials present an invaluable source for the future exhaustive studies of the scholar's still vital legacy. All these drafts, sketches, and notes await a detailed description, and obviously many of these remains require a critical edition. (...) Obviously this sketched treatise (On Phonetics) needs to be published completely and carefully, and the modest aim of the present note is merely to bring to the attention of linguists an unknown, yet remarkable further step taken by the glorious comparatist after his Mémoire, but especially to offer a preliminary extract from Saussure's systematic outline of his original attitude to the basis questions of general phonetics; it is noteworthy that in some respects the views of this outline differ from the Saussurian statements hitherto available to the readership " (je souligne) ${ }^{2}$.

2 Si l'on laisse pour un moment de côté la question de savoir si les manuscrits de Harvard modifient substantiellement notre compréhension de Saussure, on est obligé de constater au moins leur importance philologique et historique ${ }^{3}$. Ils couvrent trente-cinq ans de la vie de Saussure : de 1872 à 1907. Mais surtout, ils comportent des réflexions appartenant à des intérêts disciplinaires divers. Je ne mentionne en ce lieu que les ensembles facilement isolables et en faisant abstraction des documents qui ne sont pas de la main de Saussure. Il y des dizaines de notes et de fragments parfois notés sur des bouts de papier, des feuilles de tout format ou des enveloppes. Facilement repérable et isolable est l'Essai pour réduire les mots $d u$ grec, du latin et de l'allemand à un petit nombre de racines ${ }^{4}$, document charmant bien connu depuis qu'il a été publié : c'est comme si tout le génie de Saussure 
était déjà déposé dans cet écrit juvénile. Forment également un tout, le texte du Du génitif absolu en sanskrit, thèse de doctorat soutenue à Leipzig (écrit à la main dans un cahier et incomplet puisque plusieurs pages ont été découpées) et les vingt Cahiers de recherche qui montrent comment Saussure a préparé sa thèse. Ces documents témoignent d'une grande maitrise philologique sans avoir de l'importance méthodologique ou conceptuelle. Il faut mentionner encore les réflexions de Saussure plus ou moins systématiques concernant certaines langues, l'arménien, le lituanien, le grec, le latin et surtout, de toute évidence, le sanskrit. L'indo-européen, également, est omniprésent. Saussure propose la description de certaines spécificités grammaticales (en syntaxe et en lexicologie) et il se lance même dans des considérations sémantiques concernant ces langues. Mais les deux domaines d'intérêt les plus fréquemment traités dans les manuscrits de Harvard sont la phonétique et la mythographie. J'en dis maintenant un mot.

3 Les notes sur la phonétique sont regroupées essentiellement, mais pas complètement, dans les cinq cahiers de la Chemise 8. Ce sont bien ces notes (au moins 177 pages) dont Jakobson trouvait la publication hautement souhaitable. On y trouve au moins deux tables des matières provisoires, des pages qui ne comportent que des têtes de chapitre, des notes de lecture plus ou moins systématiques (par exemple, de textes d'Osthoff, de Brugman et de Sievers), un texte qui ressemble fort à une introduction avec une délimita tion du domaine de recherche, des chapitres entamés et d'autres plus développés. Il me semble qu'il faut situer et comprendre ces notes sur la phonétique en les lisant conjointement avec les Conférences à l'Université de Genève de 18915, avec les Notes inédites de F. de Saussure (datant d'avant 1900, donc d'avant le Premier Cours [dans la copie faite par Sechehaye]) et même au Cours de phonétique grecque et latine de 1909-1910.

4 L'autre domaine de grand intérêt dans les manuscrits saussuriens de Harvard est celui de la mythographie. Jakobson avait classé ces matériaux essentiellement dans les neuf sections, de longueur et de valeur très inégales, de la chemise 6 (qui comporte également, et contre toute logique, la plupart des Cahiers de recherche de la thèse de Leipzig) sous le nom trop général de: Notes on Linguistics. On y trouve des remarques sur des formes sanskrites tout comme des notes linguistico-historiques sur la culture hindoue. Toutefois, l'intérêt de Saussure est bien plus large que le seul sanskrit. C'est ainsi que l'on y trouve des traductions de mythes hindous. Il développe aussi et in extenso sa conception de problèmes classiques d'indologie (par exemple, l'absence de sens historique en Inde, l'oralité des mythes védiques et l'absence de variantes), et il manifeste surtout son grand intérêt pour la mythologie védique. Bien qu'il écrive : «Je suis très peu versé dans la littérature hindoue $\|^{6}$, il n'hésite pas à prendre position dans une querelle à propos de l'interprétation des deux légendes hindoues, La mort de Valmiki et Cunacepa, que Leconte de Lisle avait proposée dans ses Poèmes antiques? ${ }^{7}$. Certains de ces textes sont de vulgarisation, comme un long exposé qu'il consacre à la pensée védique, d'autres sont plus compliqués et il y en a qui relèvent de la pure spéculation théosophique ${ }^{8}$. »

5 Il m'est impossible de traiter exhaustivement tous ces matériaux - je manque des connaissances techniques nécessaires pour embrasser l'ensemble des domaines couverts et des perspectives ouvertes par les manuscrits de Harvard. Mon point de vue de commentateur sera celui du philosophe du langage profondément intéressé par la linguistique générale. Curieusement, les manuscrits de la Houghton Library ne comportent que peu de textes qui concernent directement la conception globale du langage chez Saussure - seuls quelques fragments concernent le statut du «concept linguistique » et le problème de la «réalité linguistique ». Toutefois les manuscrits de 
Harvard offrent une vraie mine d'or qui touche indirectement la linguistique générale. La conception saussurienne du langage et du discours, du sujet parlant et du temps linguistique est sous-jacente aux fragments sur la phonétique et la mythographie. En ce qui concerne la phonétique, j'avais retenu lors de mes transcriptions les rubriques suivantes : Délimitation méthodologique du domaine de la phonétique, et surtout Vers une théorie du phonème où je distingue sept thèmes de réflexion: le son, sa forme et sa substance; la combinaison des phonèmes dans la parole; physiologie et physique du son; importance de la voix; intention et volonté; le temps du son; et théorie de la syllabe. En ce qui concerne la mythographie, j'ai utilisé les rubriques suivantes : Connaissance de l'Inde (avec, en sous-rubriques: L'Inde et l'Occident; Histoire et chronologie de l'Inde), La pensée hindoue (la théosophie, essence de la pensée hindoue, la substance et les formes, le karma, le Moi), Le Véda (éléments généraux, acteurs et personnages, questions linguistiques), et enfin Mythes et légendes (Valmiki, Cunacepa, Le dossier Leconte de Lisle) ${ }^{9}$. Me situant dans une perspective plutôt philosophique, je me suis efforcé de voir plus spécifiquement ce que les manuscrits de Harvard contiennent sur le Temps et sur le Moi : Réflexions saussuriennes sur le Temps et le Moi. Il me semble qu'ils apportent un supplément à notre connaissance de la pensée saussurienne. Les manuscrits de Harvard, en effet, développent les notions de Temps-sphère et de Moi-sommeil qui 'modifient', si j'ose dire, 'marginalement' la connaissance que l'on avait déjà de la conception saussurienne extrêmement compliquée du temps linguistique/discursif et du sujet parlant.

\section{Prolégomènes : quel Saussure transposera-t-on ?}

"Saussure est d'abord et toujours l'homme des fondements. Il va d'instinct aux caractères primordiaux, qui gouvernent la diversité du donné empirique ", écrivait Benveniste en 1963, juste cinquante ans après la mort du Maître genevois ${ }^{10}$. Saussure avait noté, déjà dans le Mémoire, que cette recherche des fondements n'équivaut pas «à des spéculations d'un ordre transcendant, mais (est) une recherche de données élémentaires, sans lesquelles tout flotte, tout est arbitraire et incertitude $»^{11}$. Il y a certainement chez Saussure ce geste démocritéen : il faut aller à travers la diversité des données empiriques vers une profondeur, et enraciner ainsi le contingent dans sa nécessité propre. Saussure avait évoqué le geste démocritéen dans le Mémoire avec une belle formule : il faut « saisir les phénomènes dans leur lien intérieur $\aleph^{12}$. Geste que Bachelard aurait pu commenter ainsi : on trouve plus dans le réel caché que dans le donné évident. Toutefois, le geste démocritéen est toujours doublé chez Saussure par un geste constructiviste : le fondement, c'est l'identité. Et l'identité, c'est un « corps de définitions ", c'est une algèbre de relations déterminantes. C'est surtout, pour Saussure, un point de vue. Aucun objet n'est donné un seul instant en soi, même pas ce qu'il y a de plus matériel, le son vocal. « Il y a d'abord des points de vue, justes ou faux, mais uniquement des points de vue à l'aide desquels on crée secondairement les choses $»^{13}$. Le point de vue " crée » la substance, le point de vue est une ascèse ${ }^{14}$, mais également une éthique. Voilà donc ce qu'il y a d'absolument essentiel au projet de Saussure, et Benveniste ne s'est pas mépris sur ce premier principe " qui présume une intuition totale du langage, totale à la fois parce qu'elle contient l'ensemble de sa théorie, et parce qu'elle embrasse la totalité de son objet $\aleph^{15}$. Les manuscrits de Harvard ne disent évidemment pas autre chose, par exemple quand Saussure évoque, à propos des racines du lexique, sa conception de la réalité du fait linguistique : 
Sans doute, dès qu'il s'agit d'une chose comme la racine, on peut dire : qu'appelezvous réel ? Mais la réponse ne peut être ... Il n'y a qu'un cinquième de réel dans les racines de lexique. Mais on pourrait dire: qu'appelez-vous réel? Quiconque propose une forme de racine veut faire oeuvre rationnelle, puisque ce n'est pas la langue elle-même qui a désigné ... (interrompu) [extrait du Fragment 21].

On trouve la même idée dans les fragments concernant la théorie du phonème :

(Tout ordre phonétique) ne peut être rationnellement compris que si l'on connaît le rapport de ce qui est et de ce qui pourrait être, en adoptant certaines limites plus ou moins justifiées comme équivalence sémiologique [extrait du Fragment 35]. La bonne partie des objets de la phonétique sont des êtres de raison, n'existent que par leur définition. Il n'est pas loisible à qui s'en occupe de parler comme si ces objets tombaient sous les sens; il doit s'entourer d'un appareil de définitions extrêmement serré [Fragment 36].

8 Toutefois, ce geste démocritéen/constructiviste ne réalise que le premier principe du 'Saussure essentiel'. Une seconde clé introduit un principe complémentaire : c'est le geste dualisateur. L'objet à étudier n'est pas seulement serré - épithète typiquement saussurien : les phénomènes 'dans leur lien interne' - mais également et en même temps double. L'ob jet est une correspondance, et la « correspondance seule est objet de science ». Benveniste formulait ainsi ce principe: «Le langage, sous quelque point de vue qu'on l'étudie, est toujours un objet double, formé de deux parties dont l'une ne vaut que par l'autre ${ }^{16}$. D'où la longue série des dualités oppositives : son/sens, articulatoire (physiologique) /acousti que, individu/société, matériel /insubstantiel, mémoriel /syntagmatique, intuitif/ discursif, identité /opposition, synchronique/diachronique. Un texte des Notes inédites formule à merveille que ce principe de la correspondance ou de la balance présuppose la dominance indisputable de l'idée de différence: "A est impuissant à rien désigner sans le secours de B... tous les deux ne valent que par leur réciproque différence... aucun ne vaut même par une partie quelconque de soi autrement que par ce même plexus de différences éternellement négatives ${ }^{17}$. Une petite annotation des manuscrits de Harvard dit pourtant: Différence terme incommode, parce que cela admet des degrés [Fragment 19]. Saussure semble donc bien hésiter sur le caractère radicalement oppositif de la différence puisqu'il évoque la possibilité d'une gradualité (" de degrés») dans les différences, comme si la différence elle-même pourrait être affectée par la qualité d'une substance. Tout un champ d'inquiétudes est ainsi suggéré dans les manuscrits de Harvard, mais il va de soi qu'elles ne parviennent pas à bousculer les gestes du 'Saussure essentiel', le geste démocritéen/ constructiviste et le geste dualisateur. L'unité primaire, c'est le couple, la paire, et la caractéristique inhérente à la paire prévaut sur celle qui distingue chaque élément au sein du couple. Il faut ajouter en dernier lieu que les deux principes eux-mêmes se présentent comme les termes d'une balance, et c'est sans doute là que réside le noeud même de la 'rationalité' saussurienne. D'une part il y aurait la reconstruction (rendue possible par le geste démocritéen-constructif), et d'autre part la comparaison (rendue possible par le geste dualisateur).

Ce serait donc dans la tension entre la reconstruction et la comparaison qu'il faudrait situer l'essence spécifique de l'épistémologie saussurienne. Une phrase du Cours ajoute explicitement que cette tension est orientée, qu'il y a une finalité dans cette relation tensive entre la reconstruction et la comparaison :

Si le seul moyen de reconstruire est de comparer, réciproquement la comparaison n'a pas d'autre but que d'être une reconstruction. Sous peine d'être stériles, les correspondances constatées entre plusieurs formes doivent être placées dans la perspective du temps et aboutir au rétablissement d'une forme unique ${ }^{18}$. 
10 Si l'on se tient à ce texte 'orthodoxe', il y aurait une finalité : la comparaison des termes oppositifs serait le moyen pour aboutir à l'algèbre des formes, pur faisceau d'hypothèses abstraites. Toutefois, il pourrait y avoir une menaçe d'incohérence si l'on promulgue la finalité ou l'orientation de la tension entre la reconstruction et la comparaison. Je dirais que cette finalité fonctionne si la comparaison concerne deux termes oppositifs substantiels . Si, par contre, les termes oppositifs sont le fruit d'une catégorisation, d'un 'feuilletage'19 entre 'ordres rationnels', il ne me paraît pas évident qu'il faille choisir pour une finalité quelconque dans la relation, entre la reconstruction et la comparaison. La comparaison, dans ce cas, n'a rien de phénoménologique : on 'compare' des ordres (par exemple, l'ordre des sons et l'ordre des sens, l'ordre intuitif et l'ordre syntagmatique). On 'compare' pour reconstruire, on 'reconstruit' pour comparer. Si l'imaginaire saussurien a quelque chose d'ailé (l'imaginaire hjelmslevien étant plutôt 'contemplatif'), selon un mot de Zilberberg ${ }^{20}$, c'est que l'épistémologie du 'Saussure essentiel' est dynamique - elle se déploie comme une tension, elle incorpore la césure, la déhiscence, sans être orientée par une fin, ce qui signifierait la résolution des balances et des correspondances et l'établissement d'une épistémologie statique. On retrouvera des éclats de cet imaginaire ailé tout au long de notre parcours à travers les manuscrits saussuriens de Harvard. Les problèmes d'interprétation sont multiples et complexes, et les fragments des manuscrits de Harvard, plus encore que les notes déjà publiées, ne sont qu'un canevas "sur lequel on peut broder »- pour employer une belle expression de Engler ${ }^{21}$. C'est une aventure risquée, et une déviation due à une Hineininterpretierung n'est pas inimaginable.

\section{La physique du temps-sphère}

11 Notre premier thème est celui du Temps. Les manuscrits de Harvard évoquent ce thème fréquemment en relation avec la théorie du phonème. C'est la notion de physique qui nous permettra de faire le pont entre la théorie du phonème et le thème du Temps.

\section{L'oreille et son contexte}

12 La phonétique - phonétique générale, ou mieux : phonétique sémiologique - est définie lapidairement dans les manuscrits de Harvard par l'équivalence suivante, qui nous servira de fil rouge dans cette investigation : Phonétique = acoustico-physico-physiologique [Fragment 23].

13 La discussion concernant le statut épistémologique de la théorie du son et du phonème était, de toute évidence, dans l'air à la fin du siècle précédent. Saussure est très présent dans ce débat. Joseph Vendryès résume la position "officielle » de Saussure dans son compte-rendu du Cours :

La doctrine de F. de Saussure diminue singulièrement l'importance de la phonologie en linguistique. Ce phonéticien subtil, auquel on doit d'excellentes analyses faites sur les parlers vivants, ne craint pas d'enseigner que, quand on a expliqué tous les mouvements de l'appareil vocal nécessaires pour produire chaque phonème, on n'a éclairé en rien le problème de la langue. La phonologie, qui ne relève que de la parole, est à ses yeux une discipline auxiliaire, accessoire de la linguistique, science de la langue. Même le rapport qui existe entre la langue et la parole n'est pas phonolo gique : il est acoustique. Quelle vue originale et féconde! F. de Saussure subordonne le phénomène phonétique au phénomène acoustique. C'est par l'oreille que le sujet parlant a acquis la langue ; l'image initiale imprimée en son cerveau est une image 
acoustique. La phonation n'est que l'exécution des images acoustiques. Par suite, l'image musculaire de l'acte phonatoire est secondaire; et l'étude du mécanisme de l'articultion peut rester en dehors de l'étude de la langue. Ainsi libérée de ses attaches avec la physiologie, la linguistique ne tombe pas pour cela dans la psychologie. Son domaine propre est à égale distance des deux. Sans doute l'empreinte que laisse dans le cerveau l'image acoustique est essentiellement un fait psychique. (...) Mais l'élément psychique peut être mis à part de la langue autant que l'élément phonologique. Le linguiste n'a pas à tenir compte du travail psychique préalable à la langue qui est supposé accompli pour que la langue existe. L'acte linguistique est dans l'association d'un concept psychique et d'une image acoustique... Science de rapports entre des valeurs, la linguistique fait partie des sciences sémiologiques ${ }^{22}$.

Ce résumé de la position de Saussure que Vendryès présente en toute fidélité, donne à l' acoustique un privilège certain, et situe le statut du fait linguistique au niveau du sémiologique, le physiologique tout comme le psychologique étant tenus à distance. Cette discussion était vive parmi les linguistes de cette époque, et il faut concéder que, vers 1880, Hermann Paul semblait avoir la vue la plus synthétique sur cette problématique, surtout en ce qui concerne les régularités constatées dans le domaine du changement phonétique ${ }^{23}$. Paul énumère les traits essentiels suivants: les mouvements des organes phonateurs suscités par l'excitation des nerfs moteurs et par l'activité musculaire qu'ils déclenchent; le « sentiment kinesthésique » ou la série d'impressions dont s'accompagnent nécessairement ces mouvements; les impressions sonores qui se produisent chez les auditeurs (ces impressions ne se réduisent pas au seul plan physiologique, elles renvoient également à des processus psychologiques). Après la disparition de l'excitation physique persiste un effet psychique, appelé "images mémorielles ", entretenant un lien causal avec les processus physiologiques. Toute cette opération, en grande partie causale, n'est évidemment pas consciente: les sons sont perçus et produits en dehors de toute conscience claire, bien qu'il y ait un rigoureux contrôle, une grande capacité de discrimination de la part du sujet parlant. Et remarquons que Paul a la conviction profonde que l'image phonétique n'est pas représentationnelle (position antichomskienne et anti-cognitiviste, dirais-je) mais "qu'elle se règle sur ce qu'on entend chez ceux avec lesquels on est en communauté d'interaction ${ }^{24}$. Saussure, on n'en doute pas, ne pourrait pas apprécier cette conception synthétique de Paul, pour plusieurs raisons: Paul donne trop d'importance à la composante psychologique (les images mémorielles) et à la composante socio-pragmatique (l'impact de la communauté d'interaction), il introduit en plus du causalisme, même dans la relation du feedback psychique sur le physiologique, et il pose au coeur des régularités phonétiques le sentiment kinesthésique, c'est-à-dire la corporéité dans ses multiples virtualités sensorielles 25 .

Si on compare les intuitions de Saussure à cette vue synthétique de Hermann Paul, où toutes les composantes - physiologique, psychologique, kinesthésique, psychique tombent bien en place, on ne peut que constater un rétrécissement théorique dont il ne faut évidemment pas nier d'emblée la pertinence et l'adéquation. Mais les Notes inédites (de 1954), les Sources manuscrites tout comme les fragments très importants du Fascicule 4 n'offrent précisément qu'un canevas, ce qui nous force de combler les vides, de rendre explicite ce qui n'est que suggestif, de résoudre les contradictions, voire les paradoxes. Et les manuscrits de Harvard, dans toute leur spontanéité et leur incomplétude, rendent la tâche encore plus dure. Revenons donc au point de départ qu'est l'adéquation: Phonétique = acoustico-physico-physiologique [Fragment 23]. 
16 Il s'agit de constituer une phonétique sémiologique. Ce qui intéressera le 'phonéticien sémiologue' est l'équivalence sémiologique ${ }^{26}$. Cette phonétique ne peut se faire qu'en se libérant d'une certaine attitude 'naturelle', d'une certaine façon de parler «moulée sur

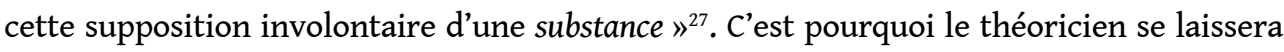
constamment interroger par la question: Qu'est ce qui est définissable? Le progrès dans la délimitation méthodique de son objet exige que l'on mette entre parenthèses' (au sens de la Reduktion husserlienne) toutes les qualifications que l'attitude naturelle nous a imposées. D’abord, il faut éliminer de la phonétique sémiologique les qualifications mécaniques, physiologiques, articulatoires:

Les intégrantes de chaque acte phonatoire: les unités d'activité musculaire, les unités de fonction acoustique, le pêle-mêle des unités d'ordres différents. (...) Il faut donc distinguer ces points de vue en tant que principe de division ou en tant que principe d'analyse, après division [Fragment 39].

17 Saussure ne nie pas qu'il faut connaître l'« appareil », la «machine $»^{28}$, et que l'on peut étudier les conditions physiologiques produisant les unités acoustiques, mais cette étude ne mènera jamais à une phonétique sémiologique, générale ou pure. C'est ce que dit pertinemment le fragment suivant des manuscrits de Harvard :

1. Dans la chaîne acoustique nous déterminons (discernons, décomposons) les moments (membres, divisions) simples, les unités homogènes (durée indifférente).. . 2. Abstrayant les moments acoustiques simples obtenus et les comparant entre eux, nous les dénombrons d'après leur qualité. ... En prenant une multitude de chaînes différentes et cela non seulement dans la langue, nous obtenons l'effectif de toutes les unités simples de qualité acoustique différente qui peuvent exister dans la parole. 3. Jusqu'ici on se meut dans la SPHERE acoustique. Nous déterminons maintenant dans quelles conditions physiologiques se produit chaque unité acoustique différente. But originaire : Pour pouvoir pénétrer l'essence de certaines transformations phonétiques se produisant au cours de l'histoire d'une langue. Mais dans la phonétique générale (PURE), le véritable objet est : avoir un moyen de définir... (phrase non complétée). 4. Opération (de) synthèse de la chaîne acoustique. Nous savons que chaque unité acoustique de qualité autre que la précédente fera un chaînon distinct, puisque c'est en analysant d'après la QUALITE que nous avons obte nu / isolé nos unités. Mais nous ne pouvons faire la synthèse de la chaîne qu'en partant du côté physiologique du phénomène; là en effet sont les barrières... [Extrait du fragment $24 ;$ je souligne et capitalise].

Ensuite, il faut éliminer les qualifications purement psychologiques, celles qui font appel à des entités ou mécanismes mentaux autonomes et pré-sémiologiques. Ce qui reste alors, c'est l'acoustique déterminant le psychique, et ce couple-là constitue un bon point de départ pour le théoricien en marche vers la phonétique sémiologique :

PHONEME opposé à SILENCE / PHONEMES individuels opposés entre eux / PHONEME par opposition à ESPECE PHONETIQUE / PHONEME par opposition à AUDITION, à SYNCHRONIE PHYSIOLOGIQUE / PHONEME par opposition à CHAINON ... Délimitation au nom de la sémiologie du phonème (négative seulement) / Délimitation acoustique du phonème (par opposition au silence) / Délimitation des causes physiologiques du phonème [Fragment 38].

19 Nous voilà en bonne route: la phonétique sémiologique ou sémiologie du phonème considérera essentiellement le phonème comme opposé au SILENCE - la délimitation acoustique du phonème par opposition au silence. Hypostase du SON, glorification de l'OREILLE - le fait phonétique nous est donné par la sensation auditive :

Le fait phonétique nous étant à son tour donné par la SENSATION AUDITIVE, c'est d'après cette dernière seule que nous fixons les actes phonatoires [Extrait du Fragment 44]. 

est la forme 'pleine', tandis que la forme sourde est 'réduite' :

Aussi est-ce en réalité la forme sonore de chaque phonème que nous [considérons] comme sa forme normale, et la forme sourde comme une réduction. (...) Il suffit de substituer/remplacer partout sonorité par retranchement de la sonorité, en posant la sonorité comme le fond uniforme/commun à tous les phonèmes [Extrait du Fragment 54].

21 L'oreille est bien présente dans la phonétique sémiologique, non pas celle du physiologiste

${ }^{29}$, mais l'oreille du sujet parlant et 'analysant' qui saisit les saillances :

L'OREILLE ne peut naturellement décider que les ressemblances, identités et différences des perceptions... [Fragment 32] ${ }^{30}$.

22 Mais qu'est-ce que saisit l'oreille analytique ? Elle 'analyse' les ressemblances, identités et différences quantitatives (c'est ainsi qu'elle saisit la différence entre une consonne et une voyelle), mais cette analyse quantitative ne devient une véritable saisie que quand le physique qualitatif est co-perçu comme la sphère dont on entend parler - les 'ressemblances, identités et différences' ne deviennent de véritables saillances que sous cette condition. C'est ce que dit l'important Fragment 48 des manuscrits de Harvard :

Différence entre consonne et voyelle est 'quantitative'. C'est ainsi qu'on fait les définitions sans s'inquiéter de la sphère dont on entend parler. Physiologiquement quantitatif, physique qualitatif (sic) [Fragment 48].

Il y a donc une sphère qualitative sur laquelle se profilent les ressemblances, identités et différences comme des saillances, et cette sphère qualitative est physique : le physique est la sphère, l'ambiance, l'horizon de l'acoustique. La phonétique sémiologique est en fait une physico-acoustique. Saussure semble suggérer qu'il y a une saisie qualitative de la sonorité par l'oreille où l'ambiance physique est co-perçue comme le contexte de l'analysandum (ressemblances, identités, différences). Toutefois, cette saisie de la qualité physique ne peut être une saisie de substances puisque la phonétique sémiologique n'a à faire qu'à des valeurs. C'est précisément sur ce point que les manuscrits de Harvard apportent du nouveau: hypostase de la sonorité, glorification de l'oreille, comme nous disions déjà, mais en plus appréciation de la qualité du physique ambiant. La qualité n'est pas substantielle mais formelle: elle fonctionne comme contexte, comme horizon de l'analysandum (ce qui est 'analysé' par l'oreille) : la saisie des valeurs place l'analysandum dans son contexte physique ambiant. Saisir un son comme une valeur, c'est contextualiser l'analysandum acoustique. Le physique est ce transcendant formel qui valorise l'acoustique c'est bien pourquoi la phonétique sémiologique est une physicoacoustique.

\section{Le Temps ambiant}

Il convient maintenant de démontrer, à l'aide des manuscrits de Harvard, que ce physique, ce transcendant formel et valorisant, a sa propre temporalité - la Temps de l'oreille qui saisit les ressemblances et différences sonores dans leur ambiance, dans leur contexte physique ${ }^{31}$. C'est bien ce Temps dont Saussure affirme dans les manuscrits de Harvard :

Le temps est pour l'OREILLE ce que l'espace est pour la vue [Extrait du Fragment 52]. 
Saussure oppose ce «Temps d'identité du phénomène physique » au «Temps d'identité de la sensation acoustique » et au «Temps d'identité physiologique » (Temps du jeu des organes) $^{32}$ :

TEMPS d'identité de la sensation acoustique; TEMPS d'identité du phénomène physique; TEMPS du nombre et de la qualité des facteurs (du jeu des organes) [Fragment 56].

On peut d'emblée faire abstraction du Temps physiologique, le temps du «jeu des organes » qui n'a aucun statut sémiologique. Restent alors le Temps ambiant ou « Temps d'identité du phénomène physique », sujet de notre étude, dans son opposition au Temps linéaire ou "Temps d'identité de la sensation acoustique ». Saussure ne mentionne pas dans ce fragment le temps dont il a parlé canoniquement dans le Cours, le temps de l'altération linguistique, Temps-facteur ou Temps externe.

Ce dernier est pourtant le plus communément accepté et le plus facile à conceptualiser ${ }^{33}$. Ce Temps dévastateur exerce sur la langue des effets: la diachronie, le changement, l'altération qui peut être d'une double nature :

«Voilà déjà de quoi faire réfléchir sur le mariage d'une idée et d'un nom quand intervient ce facteur imprévu, absolument ignoré de la combinaison philosophique, le temps. Mais il n'y aurait là rien encore de frappant, rien de caractéristique, rien de spécialement propre au langage, s'il n'y avait que ces deux genres d'altération, et ce premier genre de dissociation, par lequel l'idée quitte le signe spontanément, que celui-ci s'altère ou non. ... Ce qui est caractéristique [pour le second genre d'altération], ce sont les innombrables cas où c'est l'altération du signe qui change l'idée même » (Notes inédites, 20, 69).

Les signes sont affectés par le Temps - les effets du Temps-facteur sont irréversibles. Les Trois Conférences de Genève ont précisément comme thème ce double principe, celui de la transformation dans le temps contrebalancé par celui de la continuité dans le temps ${ }^{34}$. Toutefois, il n'importe pas au linguiste de conceptualiser ce temps : qu'il soit dit 'naturel', 'objectif', 'cosmologique' ou 'chronologique', que l'on distingue entre temps et durée ou non, cela n'a aucune répercussion sur la nature des effets linguistiques. La nature du changement - qu'il soit analogique ou mécanique - ne dépend pas de la nature du Tempsfacteur, mais du jeu des " côtés physiologique et psychique ». Le Temps-facteur est externe , il n'est pas linguistique ; ce Temps ne devrait être présent dans la théorie linguistique que comme concept primitif et non-analysable.

Bien différent est le Temps linéaire que les manuscrits de Harvard évoquent à plusieurs reprises. Il est suggéré comment la linéarité est le mode d'intervention du temps dans la parole ${ }^{35}$, voire du discours (tout comme la diachronie et le changement sont le mode d'intervention du temps dans la langue); la chaîne discursive se présente à nous forcément comme une ligne. L'identité d'une position (spatiale) sur la ligne, est-il dit, présuppose du Temps :

Ce qui équivaut à zéro n'est pas sa courte durée mais son identité avec le temps qui précède ou suit. De même ce qui fera que l'espace de son (ne) comprend plus d'unités, n'est pas sa longue durée mais la non-identité [Fragment 57].

Et cette ligne est celle de la chaîne phonétique. Les manuscrits de Harvard insistent sur le caractère concret, i.e. sonore de cette ligne temporelle :

Quand on parle de chaîne phonétique on a toujours en vue une chose concrète. Concrète s'il est conçu comme occupant un espace/une PORTION DE TEMPS. Abstraite si l'on ne parle que des caractères distinctifs, et si l'on classe. Le phonème dans la classification est une idée abstraite. Le phonème dans la chaîne phonétique 
est une idée concrète [Extrait du Fragment 45]. Chaînon: espace de son, ou un silence, ou un son que l'oreille ne juge pas identique avec lui. Il n'est pas même besoin de fixer un minimum de durée, car puisque nous ne parlons que des sensations perçues, il est clair que ce qui n'a pas pu se traduire à la sensation à cause de son infime durée est exclu d'emblée [Fragment 58].

31 Les manuscrits de Harvard ne discutent nulle part le Temps-facteur tandis qu'ils font abondamment mention, on l'a vu, du Temps linéaire. Toutefois - et c'est là qu'il fallait aboutir - on y trouve évoqué également et avec une grande force de suggestion le Temps ambiant. La façon dont ce Temps-là est présenté dans les manuscrits de Harvard, est proche de quelques textes des Recherches sur la Légende où il est dit explicitement que la notion de Temps-facteur n'est pas applicable quand on a à faire « dans la constitution même de l'être, au fantôme obtenu par la combinaison fuyante de deux ou trois idées ${ }^{36}$. Texte fascinant mais plurivoque, il va s'en dire, qui nous parle d'un troisième Temps trans cendant l'opposition classique entre temps objectif et temps subjectif, Temps-facteur et Temps linéaire. J'appelle ce troisième Temps : Temps ambiant ou Temps-sphère. Ce Temps est sphérique: non plus une ligne, mais un cercle, non pas un cercle fermé mais un cercle en propension, une 'couronne' en propension vers l'infini. Ce Temps est une ambiance, une (atmo-)sphère dans laquelle le sujet parlant « se meut », un « lieu d'existence » ${ }^{37}$. Saussure affirme, dans l'important Fragment 48, on l'a vu, que c'est à partir de ou dans la 'sphère' qu'on entend parler. Et cette sphère, c'est le physique qualitatif, dit le même Fragment, avec sa temporalité propre: «Temps d'identité du phénomène physique » [Fragment 56, cité plus haut]. Par conséquent, Temps qualitatif. C'est bien ce que dit, de manière sans doute trop allusive, un autre texte des manuscrits de Harvard :

Les unités de la chaîne sont donc fondées sur le caractère propre de la sensation pendant le temps afférent. Le fait de ne pas constituer d'unité séparée résulte pour un temps de sensation de sa différence trop peu sensible avec celui qui précède ou suit, et non pas de la courte durée de ce temps, comme on est continuellement enclin à se le représenter (sons furtifs, fugitifs, transitoires appliqué (sic) au temps et non à la qualité) [Extrait du Fragment 55].

Commençons par la dernière phrase de ce fragment pour constater que Saussure évoque le Temps linéaire ["non pas de la courte durée de ce temps », " appliqué au temps »] pour l'opposer à un autre Temps, le Temps-sphère [« et non à la qualité », en rapport avec «temps de la sensation »], et qu'il oppose ces deux Temps à propos de la temporalité des «sons furtifs, fugitifs, transitoires ». Il est intéressant de rappeler le texte de la Légende cité plus haut où le troisième Temps, écrit Saussure, est pressenti de manière privilégié dans « la combinaison fuyante de deux ou trois idées ». Temps donc de la fuite («fugitif », "fuyant »), de la transition, du furtif. Il faut absolument rendre explicite que ce Temps-là n'est pas totalement indépendant du Temps de la sensation. Bien au contraire : il se greffe sur la sensation - en fait, la sphère est acoustique [Fragment 24], elle contient les sons, le contenant étant physique et le contenu acoustique : "on se meut dans la sphère » comme un lieu d'existence. Saussure ne détache pas la 'sensation' du physique qualitatif, de la sensation (acoustique) des sons. Bien au contraire. Le lien avec la sonorité est essentiel :

Localisation dans le temps. Mais on ne localise que par rapport à la sonorité, pas par rapport à un phonème [Fragment 59].

Avoir la 'sensation' de la qualité des sons, c'est 'sentir' les sons dans leur contenant, dans leur contexte physique, c'est 'sentir' le Temps ambiant, contenant ou contexte physique du Temps linéaire. Le troisième Temps n'a pas d'existence propre, il n'existe que dans son rapport tensif avec le Temps de la chaîne sonore, tout comme la Qualité n'existe pas détachée de la sonorité (quantifiable). Tension entre le contenu et le contenant, entre 
l'acoustique et le physique, entre l'oreille et son contexte, entre le son et sa couronne, entre la ligne et la sphère, entre le phonème et son ambiance. On comprend dès à présent pourquoi la phonétique sémiologique est une physico-acoustique. Et cette tension est submergée dans des temporalités elles-mêmes en relation de tensitivité: le Temps-Qualité - Temps-Forme, pourrait-on dire - est en tension constitutive avec le Temps-Quantité de la chaîne sonore. Tension, toutefois, qui imprime de l'hétérogénéité dans la sensation du sonore. Pourquoi, pourrait-on se demander? C'est que le sujet qui fait l'expérience de l'hétérogène (qui sent la tension des temporalités dans la sonorité), est, selon les manuscrits saussuriens de Harvard, un Sujet Logique, un Moi-sommeil. C'est ce que je me propose de démontrer dans la seconde partie de ce travail.

\section{La carriere du moi-sommeil} pas l'organe physiologique puisque tout «mouvement physiologique» a été repoussé systématiquement en dehors de la portée de la phonétique sémiologique. L'Oreille n'est pas l'esprit non plus en tant qu'ensemble d'états mentaux ou psychologiques autonomes et pré-discursifs. Le psychologique n'est pas couvert par la phonétique sémiologique (dont l'objet n'est pas l'idée mais le son/idée). Qui est ou qu'est-ce que c'est que l'Oreille? C'est la 'faculté' en nous qui saisit le physico-acoustique et sa temporalité. Ainsi l'Oreille est-elle l'analyste contextualisateur sans imposer ses idiosynchrasies individuelles à l'univers sonore, elle n'est ni subjective ni objective. Elle détecte les ressemblances et les différences acoustiques contre cet horizon physique ambiant qu'est la donation sonore. Elle désobjectivise la chaîne sonore, elle désubjectivise la projection d'une ambiance. De même pour les temporalités marquant les deux pôles, la chaîne sonore et son horizoncontexte: elle désobjectivise le Temps linéaire, elle désubjectivise le Temps-sphère. L'Oreille est ainsi l'analyste désobjectivé sans devenir pour autant subjectif, et le contextualisateur désubjectivé sans devenir pour autant objectif. On est, de toute évidence, dans le royaume des troisièmes termes: ni objet ni sujet, l'Oreille est un Tiers que le Grand Dichotomisateur Ferdinand de Saussure ne sait penser que par approximation et en sombrant souvent dans le paradoxe. Les manuscrits de Harvard offrent deux pistes qui me semblent originales et inconnues. Saussure forge deux théorèmes qui donnent une certaine substance au Tiers qu'est l'Oreille ou l'analyste contextualisateur. D'abord, il va en parler en termes modestement modaux : l'Oreille est une Intentionalité indirectement intentionnelle, une Volonté indirectement volontaire. Ensuite, en termes 'mythographiques', l'Oreille sera dite en sommeil, dans un état entre l'éveil et le rêve ; l'Oreille, ce sujet-objet est en fait un Moi-sommeil. J'explore dès à présent ces deux pistes.

se revéler utile d'indiquer en premier lieu, mais brièvement, comment Saussure conçoit, toujours timidement et non sans apories, dans les écrits connus (le Cours mais surtout les Sources manuscrite ${ }^{38}$ ) cette problématique du Tiers. L'analyse contextualisatrice est présente dans le corpus saussurien au moins sous la forme des notions suivantes: interprétation, discours et acte créateur, notions toutes absentes des manuscrits de Harvard. En ce qui concerne l'interprétation, il est dit dans les Sources manuscrites que « la langue est interprétée par la génération qui la reçoit» et que l'action d'interprétation «se manifeste par des distinctions d'unités ${ }^{39}$, somme toute par la saisie de ressemblances et de différences. Saussure discute dans ce texte la langue mais la description est identique à son traitement de la chaîne sonore : interpréter la langue est comme analyser la chaîne 
sonore puisque les deux aboutissent à la saisie de ressemblances et de différences. Cet acte d'interprétation est, en fait, un Interprétant, ayant le même statut théorématique que l'Oreille. Plus connue puisque éparpillée partout dans l'oeuvre' saussurienne, est l'idée de l'élasticité discursive, de la syntaxe dynamique : le discours fonctionne alors exemplairement comme un Tiers coincé entre la langue et la parole. Selon le geste dichotomisateur, il n'y a que deux domaines : si la série grammaticale est dans la langue, la combinaison (par un choix de l'individu) sera dans la parole ${ }^{40}$. Le comble de la dichotomisation consiste à dire que la langue est spatiale et visuelle, tandis que la parole ne l'est pas - la parole étant alors, je présume, temporelle et auditive: dans ce cas de forte dichotomisation, l'Oreille est radicalement repoussée dans le domaine de la parole ${ }^{41}$. Mais les choses ne sont pas toujours aussi limpides. Le discours ou son substitut peut imposer une intermédiation : le discours affirme la lien entre concepts isolés, et cette affirmation discursive est attendue par la langue ${ }^{42}$. Drôle de formulation: la langue «manifeste des attentes» tout comme elle est dite « passer son temps »...

Ces expressions frappantes se retrouvent dans les Sources manuscrites précisément en rapport avec la créativité : «L'activité créatrice ne sera qu'une activité combinatoire... La langue passe son temps à interpréter et à décomposer ce qui est en elle de l'apport des générations précédentes - c'est sa CARRIERE ». Je reviendrai sur la 'carrière', non plus de la langue «qui passe son temps à interpréter » mais du sujet logique appelé Moi-sommeil, cet Analyste Contextualisateur dont j'ai déjà pu esquisser l'ambition. On n'en est pour le moment qu'à l'Acte Créateur dont nous parlent plusieurs textes saussuriens bien connus. La 'création', on le sait, n'est jamais ex nihilo, et c'est ainsi que l'analogie a pu être considérée par Saussure "comme le principe général des créations de la langue ». L'Interprétant, le 'Discoureur', le Créateur, autant de figures que Saussure met en scène jamais à l'avant-scène de sa théorie de base, il convient de le noter - pour capter la dynamique interne du fait linguistique.

\section{Une volonté indirectement volontaire}

Ce détour nous a confronté avec trois (pseudo-)'ouvertures' saussuriennes: acte d'interprétation, discours (syntagmatique, syntaxe), activité créatrice. Il y en a d'autres que l'on aurait $\mathrm{pu}$ exploiter: activité de classement, de comparaison, de coordination. Retournons maintenant aux manuscrits de Harvard en reprenant le fil conducteur de notre cheminement. Comment penser l'Analyste Contextualisateur qu'est l'Oreille? Saussure nous offre une première piste dans des passages d'une extrême difficulté et souvent contradictoires où il invoque les modalités de l'intention et de la volonté. L'Oreille est-elle un réseau modal combinant un vouloir et une intentionalité ?

En ce qui concerne la Volonté, le Cours et les Sources manuscrites ne laissent aucun doute : la volonté est individuelle, elle ne traverse que la parole.

Combinaisons individuelles (phrases), dépendant de [la] volonté de l'individu [D 209, EC I, 57]; Est de l'Individu, ou de la Parole: a) Tout ce qui est Phonation, b) Tout ce qui est combinaison - Tout ce qui est Volonté - Dualité : PAROLE / Volonté individuelle // LANGUE / Passivité sociale [N 22, 1, EC I, 42].

C'est d'ailleurs dans ce contexte que Saussure évoque la "nécessité de deux Linguistiques ", une linguistique de la parole et une linguistique de la langue. Et le Cours ne dit pas autre chose : 
La parole est (...) un acte individuel de volonté et d'intelligence dans lequel il convient de distinguer : les combinaisons par lesquelles le sujet parlant utilise le code de la langue en vue d'exprimer sa pensée personnelle [Cours, 30-31]; Le signe linguistique échappe à notre volonté [Cours, 104].

Le vouloir tout comme l'intelligence, autre modalité - épistémique, cette fois-ci - sont bien explicitement exclus du domaine linguistique : il est dit que l'acte linguistique n'est pas un acte de notre volonté, qu'il n'est marqué ni par la méditation ou la préméditation, ni par la réflexion - l'acte linguistique est un acte impersonnel :

Les faits linguistiques peuvent-ils passer pour être le résultat d'actes de notre volonté ? (...) De tous les actes qu'on pourrait mettre en parallèle, l'acte linguistique, si je puis le nommer ainsi, [a le caractère d'être] le moins réfléchi, le moins prémédité, en même temps le plus impersonnel de tous $[\mathrm{N} 1.1 .18, \mathrm{II}, 6]$; Du côté interne (sphère langue) il n'y a jamais préméditation ni même de méditation, de réflexion sur les formes, en dehors de l'acte, de [l'occasion] de la parole, sauf une activité inconsciente, presque passive en tous cas non-créatrice : l'action de classement [I R 2.25, EC I, 389].

Si on fait abstraction de la (pseudo-)ouverture que constitue l'action de classement, " presque passive ", donc partiellement active mais inconsciente, on voit que l'ensemble des modalités (épistémiques comme l'intelligence, la [pré]méditation, la réflexion, tout comme érotétiques comme la volonté et l'intentionalité) sont repoussées en dehors du domaine récupérable par la science linguistique: l'acte linguistique - et, par extension, l'acte phonatoire - n'est pas modalisé, ni même par ce qui toujours considéré comme intrinsèque à n'importe quel acte : l'intention. L'acte linguistique est, en fait, plutôt une activité qu'une action.

Toutefois, la pensée de Saussure, en ce qui concerne la modalisation de l'acte linguistique, est d'une complexité effrayante ${ }^{44}$. René Amacker ${ }^{45}$ a harmonisé les suggestions saussuriennes à ce propos. La volonté n'est ni de l'ordre de la langue ni nécessairement de l'ordre de la parole. D'abord, elle n'est pas de l'ordre de la langue: «[la volonté] n'intervient pas dans la création potentielle qui est la créativité syntaxique inhérente à la langue ». Ensuite, «la création à proprement parler dépend de la volonté individuelle, des nécessités externes de la communication et de l'adhérence recherchée ou non à l'égard du réel extralinguistique, etc. » Toutefois, on peut se demander si on peut attribuer cette volonté combinatoire subjective à la parole ? C'est ici qu'intervient le Troisième Terme médiateur. Il y a cette faculté mystérieuse appelée faculté de coordination qui sert à donner un ordre à la langue, à combiner les éléments selon la syntaxe. S'agit-il d'une 'faculté innée', comme le suggère Amacker, ou d'un simple mécanisme psychologique, on ne le saura pas - nous savons par contre que cette opération de coordination dépend d'une volonté. Un petit fragment repris du Cours résume le champ conceptuel : «Il n'y a pas de préméditation [volontaire], sauf - si l'on veut - dans le langage intérieur " [2526B, Cours, 227]. Primo : la volonté n'a pas d'impact sur la langue et sa créativité syntaxique ; secundo : il y a une volonté individuelle, psychologique, responsable de la 'création à proprement parler'; tertio : il y une volonté du langage intérieur, modalisation nécessaire du Tiers. Ici plus encore que pour les autres dichotomies saussuriennes, «il y a quelque chose de délicat dans la frontière des deux domaines » [D 266, EC I, 2]. Je conclus cette discussion générale. En toute conséquence, il y a une volonté - une modalisation - du langage intérieur , « limite délicate » entre langue et parole, que l'on ne saurait qualifier d'individuel ou de psychologique. C'est précisément la Volonté de l'Oreille, c'est la base modale de toute analyse contextualisante. 
linguistiques ou phonatoires: il y en a dont la réalisation est possible tandis que la réalisation de certaines autres se révèle impossible. Il convient, par conséquent, en phonétique sémiologique de classer les modalités selon leur (im)possibilité de réalisation/ manifestation. Cette posibilité ou impossibilité est indépendante de la volonté/ intentionalité.

(...) La théorie des combinaisons de phonèmes ne peut être autre chose qu'une discussion de possibilité ou d'impossibilité; la constatation et la description des combinaisons existantes ne donne qu'une vue bornée [Extrait du Fragment 46]. Lorsqu'on dit d'une combinaison de sons qu'elle est impossible, cela signifie qu'en essayant de la produire, on produit autre chose. Or si l'on ne décrit pas cet 'autre chose', on ne peut juger des causes et des limites de cette impossibilité, ni par conséquent de la possibilité contraire. Il faut fixer quelle combinaison se produit dans cet essai infructueux à la place de la combinaison voulue. Mais ici surgit une énorme difficulté, parce qu'on touche au domaine des changements phonétiques et aux questions qui dépendent de la prononciation propre à chaque langue, tandis que le sujet qui nous occupe porte uniquement sur les lois générales et absolues de l'organe humain. Plusieurs combinaisons différentes peuvent naturellement toujours remplacer celle qui est impossible, et, pour ne pas sortir de notre ordre d'idées, il faudrait déterminer laquelle est la plus indépendante de notre volonté [Fragment 29]. En phonétique, possible et impossible n'ont jamais de sens que par rapport aux unités librement définies qu'on adopte [Extrait du Fragment 42].

3. Il y a une double contrainte sur la possibilité de la réalisation des modalités. La première contrainte est mécanique et factice - certains enchaînements sont physiologiquement impossibles.

Nous sommes forcés de poser la question entre ce qui est et ce qui pourrait être au lieu que nos formules (et toutes celles qu'on pourrait inventer) n'auraient une valeur que si elles exprimaient le rapport entre ce qui est et ce qui a été... en 
spéculant sur ce qui pourrait être, c.à.d. en mesurant la latitude que laissent dans chaque cas particulier les conditions mécaniques de l'enchaînement des phonèmes, nous sommes encore forcés de nous fixer à nous-mêmes une limite factice [Extrait du Fragment 41]. Ce sont les faits plus ou moins volontaires, précisément ceux qu'on pourrait taxer de mécaniques, qui obligent de la mettre au nombre des termes en présence. (...) Il n'y a pas d'actes purement mécaniques, seulement des actes indirectement volontaires, puisque chaque fait involontaire en lui-même est la conséquence d'un fait voulu ou la condition d'un fait voulu [Extrait du Fragment 55]. Les nuances se repoduisent néanmoins dans la prononciation, parce qu'elles résultent mécaniquement $\mathrm{du}$ jeu des organes dans l'exécution des sons voulus, c.à.d. opposés. Mais elles ne sont pas dictées [Extrait du Fragment 55].

49

a seconde contrainte, théoriquement plus importante, est physico-acoustique. Il n'est

pas le cas que n'importe quelle manifestation modale soit physico-acoustiquement possible.

Dès qu'il y a un choix possible, le factum qui détermine ne peut être cherché que dans le choix d'un certain type acoustique. Il faut donc se demander dans quels cas un seul type acoustique était possible par le fait d'une seule possibilité mécanique et dans les autres cas, quel a été le type acoustique dirigeant [Extrait du Fragment 40].

5. Il est méthodologiquement intéressant de constater que certaines réalisations sont involontaires. Si B se réalise au lieu de A voulu, B sera un fait involontaire. Si on veut quelque chose, il y aura toujours une réalisation, mais pas nécessairement celle qui est voulue; dans ce cas on réalise un fait involontaire.

L'étude des faits involontaires est le chapitre où il devient nécessaire de mettre en compte la volonté, parce qu'il faut qu'on voie de chaque acte particulier s'il est volontaire ou non, et dans le dernier cas de quelle volonté (acte volontaire) il est l'indirecte conséquence (indirecte avant, pour la logique, non pour l'oreille) [Extrait du Fragment 55].

51 Le second mouvement de l'argumentation saussurienne consiste à rattacher la modalisation (l'intention, surtout la volonté) à la structure même de la sensation physicoacoustique. Il distingue trois aspects pour les unir en même temps intimement (je présente en ce lieu un bricolage que j'ai fait à partir de séquences du Fragment 55).

Trois choses si intimement unies qu'on pourrait les dire synomymes... 1. Degré de netteté de la sensation par rapport à celle qui précède ou suit... [Reprise :] ... fait qu'il n'y a pas opposition entre les sensations. 2. Individualité de la sensation/ Individualités composantes, qui fait qu'elle constitue l'unité (...) ou qu'elle ne constitue pas d'unité propre (on est tenté de rapporter au temps qu'elle occupe son individualité). D'où il résulte que le nombre des éléments à distinguer d'un mot est en raison contenu dans les qualités successives du son... [Reprise :] Les unités de la chaîne sont donc fondées sur le caractère propre de la sensation pendant le temps afférent... 3. Composition de l'image intérieure qu'on reproduit en parlant, ou nombre et nature des éléments voulus. (...) Si la qualité d'un temps de sensation ne le désigne pas pour faire unité, pour être une composante propre, cette qualité ne figure pas dans l'image voulue. L'image voulue ne contient que des oppositions. Les unités sont voulues dans la mesure où elles sont distinctes/aperçues/reconnues à la sensation... [Reprise :] L'image voulue est formée des composantes/correspondances ainsi établies. (...) C'est ainsi que la part des faits involontaires dans la prononciation se trouve circonscrite aux effets distincts, parce que s'ils étaient distincts ils deviendraient aussitôt volontaires se trouvant enregistrés dans la sensation [Extraits du Fragment 55].

La volonté ne peut être qu'indirectement volontaire -la sensation médiatise la volonté. Elle est ainsi soumise à la double temporalité : le Temps linéaire (le «temps afférent » de la chaîne acoustique) et le Temps ambiant (la « qualité » - physique, dirais-je - « d'un temps de sensation »). Ce n'est qu'à ce niveau de plénitude physico-acoustique que le Temps agit 
comme Qualité. La volonté indirectement volontaire "se compose", selon le mot de Saussure, comme une image intérieure. En toute conséquence, la volonté indirectement volontaire est la Volonté de l'Oreille. Cette Volonté est «l'image intérieure » de l'Oreille. Elle est l'analyse contextualisante intériorisée, l'image intérieure de l'Analyste Contextualisateur. La Volonté de l'Oreille n'est pas la volonté d'un sujet énonçant puisque, à l'encontre d'une subjectivité pleine, elle est domptée, contrainte par la possibilité: est possible, le réalisable; est réalisable, ce qui est inscrit dans la structure de la sensation physico-acoustique. L'Oreille n'est pas un Sujet Enonçant. Si sujet il y a sur cette dimension du Tiers, il faudrait dire que l'Oreille est un Sujet logique. Comment penser le Sujet 'logique' d'une analyse contextualisante?

\section{Le Moi-sommeil et sa carrière silencieuse}

53 Je qualifie le sujet de l'analyse contextualisante de 'logique' puisque ce sujet n'est pas le sujet énonçant, producteur des actes linguistiques, ni le sujet psychologique, extérieur et autonome à l'égard des chaînes sonores du langage. Ce n'est pas le sujet 'collectif' non plus, ce "social qualitatif», selon l'expression de Engler, qui est souvent identifié par Saussure à la langue elle-même. Est-ce un sujet transcendantal, non-empirique et pure condition de possibilité ? Non plus, puisque c'est le Sujet du " langage intérieur ». N'étant pas transcendantal, le Sujet est plutôt 'logique' - il est une règle objective métempirique (universellement observable): métempirique puisqu'il est le Principe même d'une discipline empirique, la phonétique sémiologique. Saussure est bien à la recherche d'un sujet non-subjectif qui peut fonctionner comme règle métempirique. Le Maître de Genève ne le formule pas ainsi puisqu'il ne dispose pas d'une épistémologie capable de formuler le statut du Tiers.

Confronté à la difficulté de penser en tant que linguiste le Sujet logique, Saussure se tourne vers d'autres paradigmes (Anagrammes, la Légende) et d'autres cultures (de là son intérêt pour la théosophie et le brahmanisme). Ses 'crises' manifestent des inquiétudes théoriques qui ont trait à l'impossibilité de formuler le statut du Sujet logique. Deux de ces 'crises' sont codifiées par les biographes de Saussure : 1893-4, l'année de la lettre à Whitney où il exprime des doutes quasi insupportables concernant la possibilité d'une sémiologie et d'une linguistique, et 1906, l'année du voyage de Rome et de l'impact des Anagrammes. Il est intéressant de noter qu'à chaque fois Saussure se dit impressionné par certaines spécificités mythographiques et philosophiques de la littérature védique ${ }^{46}$. Pour lui, l'hindouisme semble représenter cette marche vers le vide qui lui permet de saisir le mécanisme de la purification sémantique.

On se souvient que, dans la lettre à Whitney, Saussure présente déjà des remarques consacrées à Agni/Helios, reprises d'ailleurs dans les études sur les Anagrammes, et présentes également dans les manuscrits de Harvard. La portée de la discussion sur Agni/ Helios consiste précisément dans l'exemplification, si j'ose dire, d'une méthodologie de la vidange. Je distingue ainsi dans ce débat concernant Agni/Helios trois niveaux de pertinence. La question globale se pose ainsi : comment les hymnes védiques peuvent-ils être remplis de noms de dieux, tandis que leur conception de l'univers n'est même pas religieuse ? Saussure critique, d'abord, l'anthropomorphisation des divinités et même des figures légendaires védiques: c'est le point central du "dossier Leconte de Lisle» [Fragments 114-117 des manuscrits de Harvard] ${ }^{47}$ et l'inspiration essentielle de ses analyses des légendes de Valmiki et de Cunacepa [Fragments 110-113] : il faut 'vider' du 
nom des divinités et des figures légendaires tout sens anthropomorphique. Ensuite, Saussure discute "l'influence fondamentale des noms et de la langue sur la création des figures » - c'est le motif du texte consacré à Agni/Helios dans l'Extrait 16 du Fascicule $4^{48}$, texte qui date, selon Engler, d'entre 1903 et 1910. A la même époque Saussure rédige les textes des manuscrits de Harvard consacrés à Agni/Helios et le raisonnement est complémentaire : la divinité Surya, Soleil, dans le Véda, ne deviendra jamais 'personnelle' (à l'inverse de Agni, Feu, qui en a la tendance): Surya garde le sémantisme connotant le nom commun équivalent, et c'est le cas de toutes les divinités védiques qui ne sont pas des noms propres, même pas Agni, qui n'ont pas d' « histoire propre » : l'histoire de Surya, c'est l'histoire du soleil [Fragment 108 des manuscrits de Harvard]. S'il y a «influence fondamentale des noms et de la langue sur la création des figures ", c'est bien des noms communs - autre stratégie saussurienne motivée par l"évacuation' d'un contenu narratif trop substantiel. Enfin, Saussure reprend la question de Agni/Helios dans les études sur les Anagrammes dans la perspective d'une "analyse grammatico-poétique " (pour ne pas dire : analyse physico-acoustique). Il faut prendre le son à la lettre: la «superstition de la lettre », « l'attachement à la lettre » constitue, en fait, un retour radical au son, et le Nom Propre, de Agni/Helios, n'est qu'un Signifiant : Saussure dira dans la lettre à Meillet qu'il faut «river le Dieu au texte». On voit que c'est bien la 'méthodologie de la vidange' (d'abord du sens anthropomorphique, ensuite du sens narratif lié aux personnifications, enfin du signifié en tant que tel) qui rapproche deux sphères d'intérêt de Saussure : les anagrammes et les Védas. La lettre du 23 septembre à Antoine Meillet est explicite à ce propos :

« Avec ou sans confirmation que le phonisme du vers germanique était total, et non initial, il est naturel aussi de reposer la question pour les hymnes védiques. Je n'ai pas poussé très loin les recherches de ce côté, que j'avais vaguement entreprises l'an dernier. (...) Mais il reste au moins la question de l'anagramme qui peut s'examiner indépendamment du reste, dans le Véda comme ailleurs. Or, il n'y a pour moi aucun doute - ou pas davantage que pour le Saturnien latin - que la poésie védique est littéralement tapissée d'anagrammes, et que le poète n'avait presque rien fait quand il s'est borné à mettre ses syllabes dans une forme métrique, le principal pour lui étant probablement d'insérer le nom des dieux ou le nom des donateurs dans des cryptogrammes du vers. (...) Les neuf dixièmes des hymnes que j'ai parcourus au point de vue du nom de la divinité qui est invoquée fournissent ce nom d'une manière non équivoque et quelquefois par des imitations qui sautent aux yeux. (...) Il est probable que les différents jeux phoniques de la versification sont partis de l'anagramme, qui n'est plus qu'un de ces jeux à la fin. En effet on com prend l'idée superstitieuse qui a pu suggérer que pour qu'une prière ait son effet, il fallait que les syllabes mêmes du nom divin y fussent indissolublement mêlées : on rivait pour ainsi dire le Dieu au texte... »(dans CFS, 21(1964), 113-115).

Cette 'marche vers le son' est également une marche vers le vide. La 'méthodologie de la vidange' dont j'ai esquissé les stratégies repose sur une épistémologie, une conception de la connaissance, spécifique à la pensée hindoue, qui a fasciné Saussure comme en témoignent des dizaines de pages des manuscrits de Harvard. Son intérêt substantiel pour le Mahabharata et le Bagavad-Gita transcende de toute évidence des questions locales de grammaire sanskrite. La littérature védique et surtout le brahmanisme lui apporte, dans des moments de malaise théorique et de crise profonde, des éléments d'épistémologie. Je ne cite qu'un seul texte, parmi beaucoup d'autres, où Saussure ne cache pas ses enthousiasmes :

Curieuse Singulière (rature : contradiction) inconséquence. Il s'agit de connaître, connaître pour le Salut, ... [interrompu]. (...) Or « connaître », c'est connaître et par 
définition le brahman, c'est-à-dire une substance ou un être absolu où il n'y a rien à connaitre, puisqu'on ne peut pas déclarer qu'on l'a saisie (en son propre soi ou ailleurs) qu'après être tout à fait sûr la maintenir pure de toute contingence, et de tout attribut quelconque (activité, [rature : moralité], conscience, intelligence). La perfection qui réside dans le brahman est la neutralité absolue ne permettant de prononcer que le mot d'"être" comme pour l'Espace, qui sert à tout moment quoique involontairement, de comparaison [illisible], et il est donc suprêmement paradoxal, ou enfantin, comme on voudra, de voir tant de préoccupations ardentes dirigées vers un objet de connaissance sans doute absolu mais aussi absolument indifférent qu'il est absolu. Nous/esprits occidentaux comprenons deux doctrines: Connaissez, ou n'essayez pas de connaître l'Inconnu/Inaccessible. Il n'y a rien de tel dans l'Inde [Extrait du Fragment 88, je souligne].

Ces éléments d'épistémologie brahmanique sont "suggesteurs", dirait Saussure, en ce qu'ils esquissent la façon dont on 'connaît' le Tiers. D'aucuns parmi les lecteurs seront profondément scandalisés par la manière dont j'utilise cette piste védique et brahmanique pour saisir l'essence de la pensée saussurienne. Mais j'insiste : un quart des manuscrits de Harvard concernent la pensée hindoue et la théosophie. Que personne ne m'objecte qu'il s'agit là simplement d'un à-côté sans impact sur le 'Saussure officiel'. Bien au contraire. L'insistance chez Saussure sur la constitution de l'objet scientifique se profile sur l'horizon d'une crise essentielle : la « curieuse et singulière inconséquence » Saussure rature « contradiction », ce serait de « connaître l'Inaccessible ».

Retournons, après avoir évoqué ces perspectives insupportablement fascinantes, vers le Sujet Logique - l'analyse contextualisatrice n'est-elle pas «curieusement et singulièrement » inconséquente, voire contradictoire? Les homologations que je propose à la suite sont-elles vraiment légères? Saussure a eu l'occasion d'étudier la théosophie brahmanique dans le livre de son ami et collègue apprécié Paul oltramare, L'histoire des idées théosophiques dans l'Inde $e^{49}$. Oltramare analyse atman, l'âme brahmanique, "l'inconcevable, l'insondable, l'inintelligible, l'incommensurable », dans les Upanishads, et je ne peux me retenir de citer quelques lignes que Saussure a dû lire avec une attention empathique :

L'âme est sujet de toute connaissance ; comme telle, elle ne saurait être elle-même connue objectivement. (...) En dehors de lui, rien qui voie, entende, comprenne, connaisse. C'est là l'âme, le conducteur interne. (...) L'âme est ce qui transforme les sensations en sentiments, en perceptions, en vouloirs; elle est agissante dans le rêve comme dans la veille, empruntant au monde extérieur les matériaux dont elle construit son univers. Elle est le point de jonction de toutes les fonctions physiologiques et psychiques: 'Quand l'oeil est dirigé vers l'espace, l'atman est l'esprit qui est dans l'oeil, et l'oeil n'est que l'instrument de la vision. (...) Ce qui sait qu'il entend un son, c'est l'atman ; L'OREILLE N'EST QUE L'INSTRUMENT DE L'AUDITION'.50

59 Je laisse juger le lecteur. Les manuscrits de Harvard démontrent que Saussure a été particulièrement passionné par un autre théorème de la théosophie brahmanique, subtilement analysé par Oltramare et longuement commenté par Saussure [Fragments 96-99] : la tripartition de nidra, l'âme individuelle dans la variante Sânkhya, en trois Moi : le Moi-éveil, le Moi-rêve et le Moi-sommeil. Voici ce qu'Oltramare dit du Moi-sommeil :

C'est dans le sommeil que l'âme révèle le mieux sa nature; car, si elle rêve, elle déploie toute son activité créatrice; si elle plongée dans un sommeil sans rêve, elle est en pleine possession d'elle-même: 'Quand il s'endort, l'atman prend les matériaux à ce monde, qui contient toutes choses ; il les taille, et grâce à sa lumière propre, à son éclat, il construit lui-même. Quand il dort, c'est l'esprit qui est sa propre lumière' ${ }^{51}$. 
Parmi une multitude de textes que Saussure consacre au Moi-sommeil, je ne cite que les plus pertinents :

... Doit être que le parfait moi et le parfait principe universel, se trouve dans l'homme qui dort, à condition qu'il ne rêve pas en dormant - puisque nous avons là l'image du moi susceptible d'impressions mais n'en recevant aucune, en même temps sans conscience de son propre moi. Or justement en effet ce sommeil sans rêve que nous imaginons comme une sorte d'exemple ad absurdum, constitue une des bases fondamentales... [illisible]. C'est ici que se présente un des points que je ne puis m'empêcher de croire d'une grande importance [Extrait du Fragment 97]. On pourrait caractériser comme suit le conflit fondamental entre l'Inde et notre pensée occidentale. Pour cette dernière la question s'est posée séculairement entre le moi, comprenant ses sensations, et le non-moi/ et pour l'Inde, éternellement, entre le nonmoi et le moi en excluant du moi les sensations elles-mêmes... Une des conclusions qu'on pourrait tirer de ces singulières prémisses ... est que par conséquent l'état où se trouve le moi dans l'état psychique du sommeil sans rêve doit représenter la pureté ou l'intégrité $d u$ moi, puisque c'est dans ce seul état qu'il ne lui parvient rien de «l'extérieur ». (...) Le sommeil sans rêve (nidra) est donné comme un état psychique capital... [Extrait du Fragment 99].

61 En tant que marche vers le vide, la marche vers le son - hypostase de l'Oreille - est une descente vers le silence. Puisque la carrière du Moi-sommeil - 'carrière' au double sens du terme : là d'où on retire les ingrédients, et le chemin parcouru et à parcourir - est une carrière de silences. "Curieusement et singulièrement inconséquent, voire contradictoire»? Ce Sujet Logique, désubjectivé, vidé de tout contenu, de tout sens même, Règle métempirique, ce Sujet est le Silence en tant que Principe du Son - silence d'un sommeil sans rêves, Règle d'Or gouvernant le surgissement de ces miraculeuses étincelles sonores qui fascinent l'Oreille.

62 Cerisy, 8.1992 - Venezia, 9.1993

\section{NOTES}

* Cet article est une version largement remaniée de la communication présentée au colloque. Il est reproduit ici sous une forme abrégée, l'essentiel des notes ayant été supprimé. La version complète doit paraître dans un numéro des Cahiers Ferdinand de Saussure.

1. C'est Roman Jakobson lui-même qui m'a montré avec enthousiasme les manuscrits saussuriens dans la Houghton Library en 1971. Il se sentait bien responsable de ces précieux matériaux. Il avait effectué à cette date une première classification : le coffret noir comptait déjà les neuf chemises de volume très inégal, comme aujourd'hui. La numérotation en bas de page (au crayon) a été ajoutée dans les années quatre-vingt, sans doute par Boyd Davis. Jakobson m'exprimait lors de cette première visite à la Houghton Library en 1971 déjà son souci de ne pas trouver de chercheurs intéressés au dépouillement et à une éventuelle publication de ces manuscrits : il me disait que personne aux Etats-Unis n'avait montré de l'intérêt et qu'il cherchait en vain en Europe des candidats pour ce travail. J'ai moi-même fait un premier inventaire pendant l'été de 1971 et je suis fréquemment retourné à la Houghton Library lors de visites à Cambridge. L'existence de ces manuscrits a eu pour moi pendant longtemps quelque chose de mythique, et je ne cache pas avoir eu d'incontrôlables fantasmes concernant « cet inépuisable trésor ». Ce n'est 
que pendant l'été 1992 (juin et septembre) que j'ai eu l'occasion de faire le dépouillement systématique et la transcription exhaustive de tous les matériaux qui m'intéressaient direc tement et qui sont publiés dans les Cahiers Ferdinand de Saussure, 1993, sous le titre "Les manuscrits saussuriens de Harvard ».

2. R. Jakobson, « Saussure's Unpublished Reflections on Phonemes ", Cahiers Ferdinand de Saussure, 1969, 26, 5-14.

3. L'importance quantitative des manuscrits de Harvard n'est pas négligeable du tout. Il s'agit d'un ensemble de 639 feuilles ou 995 pages classées dans les neuf chemises du coffret.

4. L'Essai a été publié entretemps par Boyd Davis dans CFS, 32(1978), 73-101, et commenté par Jean-Daniel Candaux, "Ferdinand de Saussure linguiste à quatorze ans et demi ", CFS, 29 (1974-1975), 7-12. Voir également ce que Saussure lui-même en dit dans ses Souvenirs, CFS, 17 (1960), 12-25, surtout 16 où il qualifie son Essai d'« enfantillage ». Marie-José Reichler-Béguelin commente l'Essai juvénile dans son «Des formes observées aux formes sous-jacentes ", Présences de Saussure, Genève, Droz, 1990, 21-37 : elle y voit déjà éclore toute la 'méthode' saussurienne, explicitée évidemment dans le Mémoire.

5. 3283, 3284, 3285, dans l'édition critique de R. Engler, édition du Cours de linguistique générale, Tome 2, Fascicule 4, Wiesbaden, Otto Harrassowitz, 1974 (je renverrai, dans la suite de mon étude, aux textes rassemblés par Engler dans ce fascicule, en mentionnant le numéro d'identification de Engler et la pagination des manuscrits de Genève).

6. f12,Chemise 6, Section 1.

7. Leconte de Lisle avait réinterprété dans ses Poèmes antiques des mythes védiques, comme Surya, et des légendes hindoues, comme La mort de Valmiki (Poème 4) et Cunacepa (Poème 6), et le très long Bhagavat (Poème 3). Saussure est extrêmement dur avec Leconte : $Y$ a-t-il dans les hymnes védi ques quelque modèle que Leconte a imité dans Surya? Aucun. Le tout ressemble à une pure farce de Leconte (Fragment 116). Pour le Bhagavat, il conteste les traductions (Burnouf et Langlois) utilisées par Leconte. En ce qui concerne les deux légendes hindoues, Saussure prétend que Leconte n'a rien compris au Panthéon hindou: Les Poèmes antiques débutent par deux pièces védiques, qui n'ont rien de védique que le nom. D'abord une hymne à Surya, le Soleil. (...) Le Soleil n'a jamais été chanté de cette manière par les poètes védiques... (Fragment 117). La critique principale de Saussure consiste à dire : Le tout (le contenu de Cunacepa) est une discussion théologique, sensiblement déplacée par Leconte qui en fait le poème tragique d'un individu (Fragment 112).

8. Voir les textes rassemblés dans la Chemise Bleue, Chemise 6, Section 2, qui contient des matériaux d'un compte-rendu du livre de Paul oltramare sur l'Inde, et des réflexions profondes sur le karman, la substance, le moi, etc.

9. Je renvoie évidemment à la publication des transcriptions dans CFS, 1993 (voir 1). Les textes transcrits sont numérotés de 1 à 117, de longueur inégale. Je les cite, dans cet article, avec leur numéro correspondant.

10. E. Benveniste, "Saussure après un demi-siècle », CFS, 20(1963), 7-21. Voir également à propos de la 'méthode' de Saussure et en relation avec l'article cité de Benveniste, l'étude de Cristina Vallini, «Continuità del metodo di Sausure », Présences de Saussure, Genève, Droz, 1990, 5-19.

11. Benveniste cite ces lignes dont il dit qu'elles pourraient servir d'épigraphe à l'oeuvre tout entière de Saussure (Ibidem, 9).

12. Mémoire, 174.

13. Dans les notes inédites: CFS, 12(1954), 57-58. Et Saussure considère que la linguistique se distingue, à ce propos, des autres domaines de recherche scientifique : «Voici notre profession de foi en matière linguistique : en d'autres domaines, on peut parler des choses 'à tel ou tel point de $v u e$ ', certain qu'on est de retrouver un terrain ferme dans l'objet même. En linguistique, nous nions en principe qu'il y ait des objets donnés, qu'il y ait des choses qui continuent d'exister quand on passe d'un ordre d'idées à un autre » (Ibidem, 58). 
14. Il faut concéder à Marie-José Reichler-Béguelin que le travail sur les anagrammes trahit chez Saussure une grande inquiétude quant à la pertinence de sa 'méthode démocritéenne' pour l'analyse de textes poétiques ou mythiques. Saussure semble bien douter de la puissance explicative de sa modélisation quand il est confronté à la créativité individuelle : « La forme sousjacente devenait dès lors une norme, un carcan, une 'mesure serrée' réglementant l'usage individuel de la parole poétique. Oscillant entre doute et certitude devant tant de tyrannie, le savant confronté aux anagrammes voyait aussi, sans doute, toute son activité encourir le soupçon de fantasmagorie" (art.cit., 1990, 35). Ceci constitue évidemment le contrecoup de l'ascèse saussurienne. L'ambiguïté de ses sentiments à l'égard de sa modélisation constructiviste apparaît dans les manuscrits de Harvard, et mon étude devrait la mettre en évidence.

15. E. Benveniste, art.cit., 15-16. Il est vrai pourtant, comme dit Marie-José Reichler-Béguelin (art.cit., 35) que «Saussure s'est refusé à mettre 'la bride sur le cou' aux données attestées : il a explicitement choisi de préserver, dans la langue, un espace pour la variation inexplicable... ».

16. Art.cit., 16.

17. CFS, 12(1954), 63.

18. Cours, 299.

19. Le mot est de Claude Zilberberg, dans « Retour à Saussure ?", Actes sémiotiques, 63(1985), 24.

20. Art.cit., 31.

21. Rudolf Engler, « Sémiologies saussuriennes ", CFS, 34(1980), 3. Engler soulève dans cet article, à propos des interprétations que Avalle a proposées des manuscrits sur la Légende, le problème général de l'interprétation des manuscrits saussuriens : «Les problèmes d'édition sont, (...), en grande partie des problèmes d'interprétation: la syntaxe et la sémantique des textes sont souvent très obscures ». Engler en tant que philologue admirable et fidèle lecteur du Maître de Genève incite à la prudence interprétative. Je suis, tout comme Avalle, très sensible «à l'image d'un Saussure énigmatique et poétique » (R. Engler, art.cit., 52) et je crois, en effet, à un Saussure " appliqué paradoxalement, en privé, à détruire ce qu'il avait publiquement érigé en dogme » ( art. cit., 51), avec cette nuance : ce 'Saussure privé' existe quelque part en écrit - entre autres, dans les manuscrits de Harvard.

22. Ce compte-rendu est repris dans C. Normand e.a., Avant Saussure, Bruxelles, Complexe, 1978, p. 168-169 (je souligne).

23. Hermann Paul, « Principes du devenir du langage » (en traduction française par P. Caussat), dans C. Normand et e.a., Avant Saussure, Bruxelles, Complexe, 1978, 51-65. Paul, dans ce texte subtil, évoque également les phénomènes de l'accent et du tempo, la variabilité et la déviation de la prononciation, la singularité du développement individuel. Voir également dans le même recueil, le texte de Hugo Schuchardt (de 1885), "Sur les lois phonétiques: contre les néogrammairiens » (trad. par P. Caussat).

24. Art.cit., 62.

25. Il est intéressant de noter que même Antoine Meillet (1893), se montre très sensible à la synthèse de Hermann Paul, en admettant que les lois phonétiques sont l'énoncé de phénomènes historiques, qui atteignent les articulations, lesquelles constituent seules des réalités physiologiques : «de ce que les lois phonétiques n'ont pas d'exception qui ne puisse admettre une explication psychologique ou historique, il résulte qu'elles sont le produit de causes inhérentes au langage d'un temps et d'un lieu déterminé » («Les lois du langage », dans C. Normand, op.cit., 97-102). Plusieurs articles de Langages 49 (1978) sur Saussure et la linguistique pré-saussurienne discutent le traitement de la relation de la linguistique à la physiologie et à la psychologie dans les théories linguistiques ambiantes à la fin du siècle précédent ; voir surtout José Medina, «Les difficultés théoriques de la constitution d'une linguistique générale comme science autonome ", 5-23. 
26. Or tout ordre phonétique [...] ne peut être rationnellement compris que si l'on connaît le rapport de ce qui est et de ce qui pourrait être, en adoptant certaines limites plus ou moins justifiées comme équivalence sémiologique [Fragment 35].

27. Extrait d'un beau texte des Notes inédites: «Comme le langage n'offre sous aucune de ses manifestations une substance, mais seulement des actions combinées ou isolées de forces physiologiques, psychiques, mentales; et comme néanmoins toutes nos distinctions, toute notre terminologie, toutes nos façons de parler sont moulées sur cette supposition involontaire d'une substance, on ne peut se refuser, avant tout, à reconnaître que la théorie du langage aura pour plus essentielle tâche de démêler ce qu'il en est de nos distinctions premières. Il nous est impossible d'accorder qu'on ait le droit d'élever une théorie en se passant de ce travail de définition » (Notes inédites 9,56 ).

28. Saussure parle dans les manuscrits de Harvard « des fonctions de l'appareil comme ceux (sic) d'une machine » [Extrait du fragment 55].

29. Dont Saussure dit dans N 14: «La meilleure preuve à donner du fait que l'impression acoustique seule a une valeur, c'est qu'il serait parfaitement impossible aux physiologistes euxmêmes de distinguer des unités dans le jeu de la voix hors des unités [préalablement fournies par] la sensation acoustique. Qu'est-ce que fait un physiologiste qui nous explique les mouvements pour $b$ ? Il commence [par] prendre une base dans l'unité que lui donne b à son oreille » [3305.7].

30. «Mais nous ne tenons compte, dans l'acte phonatoire, que de ce qui est saillant pour l'oreille, différentiel, et qui peut servir à une délimitation d'unités dans la chaîne parlée » (Sources manuscrites, I R 1.39, EC I, 133).

31. Je pense, tout comme Michel Arrivé, que les réflexions saussuriennes sur le temps ne peuvent être considérées comme marginales ni parasitaires. Le temps, par contre, est une véritable obsession pour Saussure - non pas le 'Saussure officiel' et 'standard', mais le 'Saussure polyphonique'. Comme le dit si bien Arrivé : « Dans ces subtilités et ces paradoxes se lit ce qu'est véritablement la réflexion saussurienne, quel que soit son objet apparent: une longue et obstinée méditation sur le temps » («Saussure : le temps et la symbolisation », dans ce volume).

32. Saussure note que ce dernier Temps n'a de l'importance pour la phonétique sémiotique que s'il est reconstruit méthodiquement: " Méthode qui consiste partant de l'impression acoustique à fixer/recueillir les TEMPS physiologiques ayant un intérêt direct pour la phonétique » [Extrait du Fragment 33].

33. «Le fait que le temps intervient pour altérer la langue, comme il intervient pour altérer (ou modifier) toute chose, ne semble pas d'abord un fait bien grave pour les conditions où est placée la science linguistique. Et je dois ajouter que je ne vois qu'une infime proportion de linguistes, ou peut-être aucune, qui soit disposée elle-même à croire que la question du Temps crée à la linguistique des conditions particulières, voire une question centrale et pouvant aboutir à scinder la linguistique en deux sciences » (Sources manuscrites, N 23.6, EC I 175).

34. Il s'agit de textes de vulgarisation sans aucune difficulté d'interprétation: N 1.1, N 1.2 et $\mathrm{N}$ 1.3. du Fascicule 4 [3283, 3284, 3285]. « (Nos conférences donnent) un aperçu suffisant: sur ce qu'est la condition de la langue [dans] le Temps, devant le facteur Temps; [ils nous donnent] une idée des conditions universelles où se trouve placé [un idiome quelconque] en présence du fait 'qu'un certain intervalle de temps s'écoule' - et nous nous sommes appliqués à ne faire intervenir aucun autre facteur fondamental que ce facteur de [la] durée, de la distance chronologique » [N 1.3, 3285]. On voit que Saussure ne s'intéresse pas à une détermination plus approfondie du Temps-facteur.

35. Michel Arrivé, «Saussure : le temps et la symbolisation », dans ce volume. Je dois beaucoup à cet article qui, avec compétence et imagination, jette de la lumière sur cette nébuleuse conceptuelle qu'est la 'théorie' saussurienne du Temps.

36. Je cite le paragraphe entier sachant bien que le texte s'applique à la «bulle de savon » qu'est le personnage en tant que création artistique [3958.8, 41]: "Comme on le voit, au fond l'incapacité à maintenir une identité certaine ne doit pas être mise sur le compte des effets du 
Temps - c'est là l'erreur remarquable de ceux qui s'occupent des signes - mais [...] est déposée d'avance dans la constitution même de l'être que l'on choye et observe comme un organisme, alors qu'il n'est que le fantôme obtenu par la combinaison fuyante de deux ou trois idées " (cité à partir de D'Arco Silvia Avalle, «La sémiologie de la narrativité chez Saussure », dans Essais de la théorie du texte, Paris, Galilée, 1973, 32-33).

37. Voir le Fragment 24, cité plus haut : «On se meut dans la sphère... ». On trouve un texte dans les Sources manuscrites où Saussure met explicitement en rapport sphère et lieu d'existence : «Il y deux manières, pour un mot, d'être voisin, coordonné, rapproché, en contact d'un autre : on peut appeler cela les lieux d'existence des mots, ou les deux sphères de rapports entre les mots... D'une part, il existe le trésor intérieur qui équivaut aux casiers de la mémoire; c'est là ce qu'on peut appeler le magasin; c'est un des deux lieux, une des deux sphères" (Sources manuscrites, II R 89-90, EC I, 277-81).

38. On a sans doute remarqué que j'évite systématiquement d'invoquer les Anagrammes où le problème de la subjectivité se pose pourtant de toute évidence. Giuseppe Nava écrit dans son introduction à la publication des deux lettres de Saussure à Pascoli : "Saussure voulait exclure des harmonies phoniques l'intention subjective du poète, aussi bien que l'automatisme inconscient du vers et de son schéma rythmique, pour les ramener à travers une classification logique à une règle objective métempirique universellement observée. (...) Saussure plaçait implicitement, derrière le vers, non 'le sujet créateur mais le mot inducteur' [citation de J. Starobinski, Les mots sous les mots]; il faut toutefois ajouter que, dans la mesure où l'action inductive du mot n'est pas définie selon des constantes fonctionnelles, comme elle le sera plus tard chez Jakobson, mais selon l'hypothèse d'un procédé artificiel d'origine extra-linguistique, le problème de la subjectivité se pose à nouveau, car c'est toujours le poète qui doit choisir le mot sur lequel il construit le texte grâce à un dosage exact d'éléments phoniques » (CFS, 24(1968), 74). En fait, le corpus que Saussure constitue pour ses recherches sur les Anagrammes n'est pas textuel: il s'agit de fragments oraux, sonores, dirais-je, puisqu'il s'agit de découvrir des harmonies phoniques. Je pense, par conséquent, que la notion de Temps-sphère que Saussure 'pense' (inchoativement) dans les manuscrits de Harvard est d'une grande utilité si on veut comprendre le projet des Anagrammes.

39. [Sources manuscrites, II R 101-2, EC I, 390-1].

40. Voici quelques textes souvent cités : «Ainsi une série grammaticale est bien dans la langue, mais il reste à l'individu la combinaison, laissée au choix de chacun, pour exprimer sa pensée dans une phrase. Cette combinaison est dans la parole, non dans la langue » [D 184/5, EC I, 285-6]; «La parole est l'acte par lequel l'individu réalise la faculté du langage grâce à la convention qu'est la langue » [G 1. 1b, EC I, 32, voir également D 178, EC I, 41-2 et II R 6/7, EC I, 32].

41. «La langue est tangible, c'est-à-dire traductible en images fixes comme des images visuelles, ce qui n'est pas possible pour les actes de la parole par exemple » [III C 272, EC I, 44].

42. «La langue n'est créée qu'en vue du discours, mais qu'est-ce qui sépare le discours de la langue, ou qu'est-ce qui, à un certain moment, permet de dire que [la] langue entre en action comme discours? (...) Le discours consiste, fût-il rudimentairement et par des voies que nous ignorons, à affirmer le lien entre deux des concepts qui se présentent revêtus de la forme linguistique pendant que la langue ne fait préalablement que réaliser des concepts isolés qui attendent d'être mis en rapport entre eux pour qu'il y ait signification de pensée » (Ms 3961, texte publié dans CFS, 43(1990), 93-94).

43. "Rappelons [qu'en linguistique] quand on parle de création, il ne s'agit pas de création ex nihilo. L'activité créatrice ne sera qu'une activité combinatoire, c'est la création de nouvelles combinaisons » [I R 2.77, EC I, 386] ; « Mais jamais il n'est possible à la langue de construire une forme de but en blanc [et par un acte véritablement créateur] » [N7, EC, 459]. «L'analogie n'est autre chose que tout l'ordre de phénomènes qui constituent la création incessante, journalière, dans la 
langue » [dans le Cours, 223] ; « Analogie, principe général des créations de la langue » [I R 2.19, EC I, 374]. On trouve une synthèse de cette problématique au chapitre 7.5. "Analogie et créativité » dans l'excellent livre de René Amacker, Linguistique saussurienne, Genève, Droz, 1975.

44. Je ne discute même pas la détermination toute spéciale de l'intention dans les Recherches sur la Légende. Michel Arrivé a très bien montré que «l'intention, (bien que) jamais décelable, est indispensable à l'accès au statut de symbole » (au Chapitre 1: "Le symbole en linguistique : Saussure et Hjelmslev » de Linguistique et psychanalyse, Paris, Kliencksieck, 1986, 36). Saussure parle de volonté de symbole et il y a une seule occurrence dans les Recherches où il affirme que la symbolisation se définit aisément par une mise en relation consciente et intentionnelle de deux représentations. Arrivé remarque dans une note: "On remarque le parallélisme entre deux quêtes d'intentionnalité : symbole et anagramme sont, de ce point de vue, construits de la même façon par Saussure: ils ne peuvent être posés que s'ils sont conscients et intentionnels. La possibilité même d'une structure inconsciente est récusée avec une énergie qui étonne » (op.cit., 48). Mais, même si elle est indispensable, l'intention sous-jacente à la symbolisation, n'est normalement pas décelable: l'histoire évémentielle peut permettre de repérer l'intention symbolique, mais la repérabilité de l'intentionnalité pourtant nécessaire n'est pas une conditio sine qua non. On peut conclure de ces analyses de Michel Arrivé que Saussure, à nouveau, défend une dichotomisation insoutenable: dans le domaine des signes, toute modalisation est exclue tandis que dans le domaine des symboles, la modalisation (intention et vouloir, en plus conscients) est requise comme nécessaire. Voir également le commentaire de D'Arco Silvio Avalle, op.cit., 44-46.

45. Op. cit., 203-204.

46. Qu'on se rappelle la lettre à Bally (17 juillet 1906) où l'enthousiasme de Saussure pour le védisme éclate. Il écrit à Bally qui lui avait vraisemblablement emprunté son exemplaire du RigVéda : «C'est pourquoi par parenthèse je vous demandais de m'adresser mon Rig-Veda, et c'est alors que des horizons sans fins se sont ouverts devant mes yeux " (publié par Prosdocimi et Marinetti, CFS, 44(1990), 51). En même temps Sausure déploie un intérêt très poussé pour la théosophie brahmanique et son épistémologie sous-jacente. Il prépare également le compte-rendu du livre de Paul Oltramare, L'histoire des idées théosophiques dans l'Inde (par en 1906) dont il sera question dans un instant.

47. Leconte a cru qu'il s'agissait de la situation humaine et dramatique d'une personne. Il s'agit d'une question moins humaine, mais beaucoup plus dramatique pour les gens de l'Inde, qui est celle du sacrilège ou du non-sacrilège, au point de vue théorique, ceci étant la chose suprême pour leur esprit religieux et abstrait. (...) Le tout est une discussion théologique, sensiblement déplacée par Leconte qui en fait un poème tragique d'un individu [Extrait du Fragment 112].

48. Je cite une portion de cet extrait : «Il est vrai [maintenant] que la plus vaste catégorie des êtres placés dans le Panthéon de chaque [peuple antique] provient non de l'impression faite par un objet réel, tel qu'agni, mais du jeu [infini] des épithètes roulant sur chaque nom... Ce n'est pas cela qui sera propre à nous dissuader de l'influence fondamentale des noms et de la langue sur la création de figures ». Et Saussure conclut : «Le mot est simplement déterminant : il est le premier suggesteur (sic) et la seule raison finale de la divinité nouvelle... » [N 10, 3207, Extrait 16].

49. Paris, Ernest Leroux, 1906 (il s'agit du premier volume intitulé La théosophie brahmanique) dont Saussure a fait un compte-rendu détaillé.

50. Op. cit., 78.

51. Op. cit., 79 . 